\title{
Brain Network Alterations in Alzheimer's Disease Identified by Early-Phase PIB-PET
}

\author{
Liping Fu $\mathbb{D}^{1},{ }^{1}$ Linwen Liu, ${ }^{2}$ Jinming Zhang, ${ }^{1}$ Baixuan Xu, ${ }^{1}$ Yong Fan $\mathbb{D}^{\mathrm{D}},{ }^{3}$ and Jiahe Tian $\mathbb{D}^{1}$ \\ ${ }^{1}$ Department of Nuclear Medicine, General Hospital of the Chinese People's Liberation Army, 28 Fuxing Rd, Beijing, China \\ ${ }^{2}$ National Laboratory of Pattern Recognition, Institute of Automation, Chinese Academy of Sciences, \\ 95 Zhongguancun East Road, Beijing, China \\ ${ }^{3}$ Department of Radiology, Perelman School of Medicine, University of Pennsylvania, Philadelphia, PA 19104, USA
}

Correspondence should be addressed to Yong Fan; yong.fan@ieee.org and Jiahe Tian; tianjh@vip.sina.com

Received 23 August 2017; Revised 1 December 2017; Accepted 12 December 2017; Published 8 January 2018

Academic Editor: Giorgio Biasiotto

Copyright @ 2018 Liping Fu et al. This is an open access article distributed under the Creative Commons Attribution License, which permits unrestricted use, distribution, and reproduction in any medium, provided the original work is properly cited.

\begin{abstract}
The aim of this study was to identify the brain networks from early-phase ${ }^{11} \mathrm{C}$-PIB (perfusion PIB, pPIB) data and to compare the brain networks of patients with differentiating Alzheimer's disease (AD) with cognitively normal subjects $(\mathrm{CN})$ and of mild cognitively impaired patients (MCI) with CN. Forty participants (14 CN, $12 \mathrm{MCI}$, and $14 \mathrm{AD}$ ) underwent ${ }^{11} \mathrm{C}$-PIB and ${ }^{18} \mathrm{~F}-\mathrm{FDG}$ $\mathrm{PET} / \mathrm{CT}$ scans. Parallel independent component analysis (pICA) was used to identify correlated brain networks from the ${ }^{11} \mathrm{C}$-pPIB and ${ }^{18} \mathrm{~F}$-FDG data, and a two-sample $t$-test was used to evaluate group differences in the corrected brain networks between $\mathrm{AD}$ and $\mathrm{CN}$, and between MCI and CN. Our study identified a brain network of perfusion (early-phase ${ }^{11} \mathrm{C}-\mathrm{PIB}$ ) that highly correlated with a glucose metabolism $\left({ }^{18} \mathrm{~F}-\mathrm{FDG}\right)$ brain network and colocalized with the default mode network (DMN) in an $\mathrm{AD}$-specific neurodegenerative cohort. Particularly, decreased ${ }^{18}$ F-FDG uptake correlated with a decreased regional cerebral blood flow in the frontal, parietal, and temporal regions of the DMN. The group comparisons revealed similar spatial patterns of the brain networks derived from the ${ }^{11} \mathrm{C}$-pPIB and ${ }^{18} \mathrm{~F}$-FDG data. Our findings indicate that ${ }^{11} \mathrm{C}$-pPIB derived from the early-phase ${ }^{11} \mathrm{C}$-PIB could provide complementary information for ${ }^{18} \mathrm{~F}-\mathrm{FDG}$ examination in $\mathrm{AD}$.
\end{abstract}

\section{Introduction}

The current diagnostic criteria for Alzheimer's disease (AD) include amyloid- $\beta(\mathrm{A} \beta)$ and fludeoxyglucose F $18\left({ }^{18} \mathrm{~F}-\mathrm{FDG}\right)$ positron emission tomography (PET) imaging biomarkers that provide amyloid burden and neuronal injury information [1]. Under both physiological and pathological conditions, the cerebral blood flow is coupled to cerebral metabolic rates of glucose measured by FDG-PET [2,3]. Several studies have reported that perfusion data estimated from early-phase $\left({ }^{11} \mathrm{C}\right)$-labeled Pittsburgh Compound B $\left({ }^{11} \mathrm{C}-\mathrm{PIB}\right)$, referred to as perfusion PIB $\left({ }^{11} \mathrm{C}\right.$-pPIB), correlated with glucose metabolism as estimated by ${ }^{18}$ F-FDG [4-6]. Moreover, recent PET studies using amyloid and Tau tracers indicated that early-phase images of PET tracers provided information on brain perfusion, closely related to glucose metabolism, and could be used as a neurofunctional biomarker [7-9]. Previous studies mostly focused on regions of interest or on whole brain voxel wise measurements $[5,6,10]$, which are not equipped to capture distributed variations in cross-brain networks. It remains unclear how early-phase PIB-PET and glucose metabolism correlate with each other and how such a relationship varies with disease progression at the brain network level.

Nowadays, multivariate statistical paradigms (e.g., principal component analysis [PCA] or independent component analysis [ICA]), which assess distributed variations and their interrelationships in multiple neuroimaging data, provide a better framework for the integrative analysis of multimodal imaging data. As a data-driven analytic method, ICA is a powerful tool to investigate brain networks based on neuroimaging data. This data can be collected with, including functional magnetic resonance imaging (fMRI) [11], magnetoencephalography [12], electroencephalography $[13,14]$, and 
TABLE 1: Demographic, clinical, and neuropsychological characteristics of the patients.

\begin{tabular}{lcccc}
\hline & $\mathrm{AD}(n=14)$ & $\mathrm{MCI}(n=12)$ & $\mathrm{CN}(n=14)$ & \\
\hline Sex (male/female) & $4 / 10$ & $8 / 4$ & $5 / 9$ & 0.13 \\
Age (years) & $68.1 \pm 9.9$ & $75.8 \pm 8.6$ & $67.4 \pm 5.0$ & $0.02^{*}$ \\
Education (years) & $11.6 \pm 3.9$ & $13.3 \pm 3.8$ & $11.9 \pm 4.1$ & 0.52 \\
Amyloid status (PIB +/-) & $12 / 2$ & $9 / 3$ & $1 / 13$ & $<0.001^{*}$ \\
MMSE & $19.4 \pm 3.3$ & $27.3 \pm 1.6$ & $28.4 \pm 1.2$ & $<0.001^{*}$ \\
CDR & $0.96 \pm 0.13$ & 0.5 & 0 & $<0.001^{*}$ \\
\hline
\end{tabular}

With the cerebellar cortex as the reference region, voxel wise semiquantitative calculations of global standardized uptake value ratios (SUVR) for all subjects were performed. The amyloid status was reflected by the Pittsburgh Compound B (PIB) burden and the cutoff value of SUVR was set to 1.15 to determine PIB positive or negative. Chi-square was used for the gender comparison; one-way ANOVA with a Bonferroni post hoc test was used for age and neuropsychological test comparisons. ${ }^{*} p<0.05$. AD: Alzheimer's disease; MCI: mild cognitively impaired; CN: cognitively normal; MMSE: Mini-Mental State Examination; CDR: Clinical Dementia Rating.

TABLE 2: Two sample $t$-test results of the highest correlated component pair of ${ }^{11} \mathrm{C}$-pPIB and ${ }^{18} \mathrm{~F}-18 \mathrm{~F}-\mathrm{FDG}$ between $\mathrm{AD}$ and CN and between $\mathrm{MCI}$ and $\mathrm{CN}$ groups.

\begin{tabular}{|c|c|c|c|c|c|c|c|}
\hline \multicolumn{4}{|c|}{$A D$ versus $C N$} & \multicolumn{4}{|c|}{ MCI versus CN } \\
\hline \multicolumn{2}{|c|}{${ }^{11} \mathrm{C}$-pPIB } & \multicolumn{2}{|c|}{${ }^{18} \mathrm{~F}-\mathrm{FDG}$} & \multicolumn{2}{|c|}{${ }^{11} \mathrm{C}-\mathrm{pPIB}$} & \multicolumn{2}{|c|}{${ }^{18} \mathrm{~F}-\mathrm{FDG}$} \\
\hline pvalue & $T$ value & pvalue & $T$ value & pvalue & $T$ value & $p$ value & $T$ value \\
\hline 0.0010 & -3.6907 & 0.0002 & -4.3774 & 0.0009 & -3.7993 & 0.0010 & -3.7279 \\
\hline
\end{tabular}

AD: Alzheimer's disease; MCI: mild cognitively impaired; CN: cognitively normal; ${ }^{11} \mathrm{C}$-pPIB: $\left({ }^{11} \mathrm{C}\right)$-labeled Pittsburgh Compound B; ${ }^{18} \mathrm{~F}-\mathrm{FDG}$ : fludeoxyglucose F 18.

structural MRI [15] and PET imaging [16]. Parallel independent component analysis (pICA) [17] is a variation of ICA that allows one to estimate independent components as well as multimodal patterns or mixed coefficients. pICA has recently been used to study the mechanisms by which $\mathrm{A} \beta$ deposition leads to neurodegeneration and cognitive decline [18]. It was also used to study the spatial patterns of $\mathrm{A} \beta$ deposition and glucose metabolism across an AD population [19]. Moreover, pICA has been used successfully to reveal the complex relationship between different PET elements of AD pathophysiology [19]. pICA, therefore, promises to be a suitable means of exploring the spatial patterns of regional cerebral blood flow (rCBF), evaluated by pPIB, and glucose metabolism at the level of the whole brain network.

In the present study, we adopted pICA to derive brain networks from early-phase of ${ }^{11} \mathrm{C}$-PIB and ${ }^{18} \mathrm{~F}$-FDG to explore their relationships across $\mathrm{AD}$, mild cognitive impairment (MCI), and cognitively normal $(\mathrm{CN})$ patient groups. This study was designed to (1) identify whether distinctive functional connectivity networks, such as the default mode network (DMN), can be detected from early-phase ${ }^{11} \mathrm{C}-\mathrm{PIB}$ data and (2) to explore the discriminability of the network derived from ${ }^{11} \mathrm{C}$-pPIB for distinguishing $\mathrm{AD} / \mathrm{MCI}$ from $\mathrm{CN}$.

\section{Results}

2.1. Patient Characteristics. As described in our previous study [10], patients in the MCI groups $(F=4.23, p=0.02)$ were older than in the $\mathrm{CN}$ and $\mathrm{AD}$ groups, and there was no significant difference in gender or the level of education for the two groups ( $p=0.13$ and 0.52 , resp.). Cognitive performance, estimated from Clinical Dementia Rating (CDR) and
Mini-Mental State Examination (MMSE) tests, was significantly worse in AD patients than MCI and CN participants $(F=65.93, p<0.001)$. However, no significant difference in MMSE test results was observed between the MCI and CN groups (Table 1). Furthermore, the amyloid status of all participants is shown in Table 1; the cerebellar gray matter was used as the reference region and the standard uptake value ratio (SUVR) cutoff value was set to $1.15[20,21]$.

2.2. Correlated ${ }^{11} C-p P I B$ and ${ }^{18}$ F-FDG Networks. One pair of components (networks) was identified. It had the highest correlation $(R=0.92)$ between the ${ }^{18}$ F-FDG and ${ }^{11} \mathrm{C}$-pPIB data and was largely colocalized with the DMN [22, 23]. As listed in Table 2, the highest correlated component pair also differed significantly between $\mathrm{AD} / \mathrm{MCI}$ and $\mathrm{CN}$ in their loading coefficients. This pair of components showed that a decrease in ${ }^{18} \mathrm{~F}$-FDG uptake correlated with a decrease in perfusion in the frontal, parietal, and temporal regions, including the medial frontal gyrus (MFG), anterior cingulate cortex (ACC), posterior cingulate cortex (PCC)/precuneus, superior temporal gyrus (STG), temporal pole, and orbitofrontal gyrus (Figure 1).

2.3. Group Comparisons of ${ }^{18} F-F D G$ and ${ }^{11} C-p P I B$ within the Correlated Network. Tables 3 and 4 summarize the group differences within the correlated networks in ${ }^{18} \mathrm{~F}-\mathrm{FDG}$ and ${ }^{11} \mathrm{C}$-pPIB measurements between $\mathrm{AD}$ and $\mathrm{CN}$ groups (Figure 2). Despite fewer regions detected by ${ }^{11} \mathrm{C}$-pPIB than ${ }^{18} \mathrm{~F}$ FDG in the intergroup comparisons, similar patterns were observed. The hypometabolic regions largely colocalized with the hypoperfusion areas, including the STG, Limbic lobe/ParaHippo, superior parietal lobe (SPL), PCC, and ACC. 


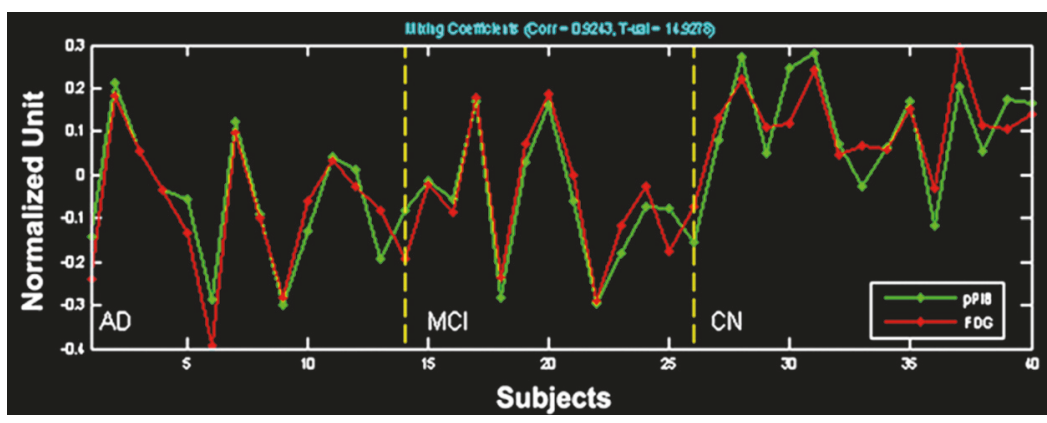

(a)

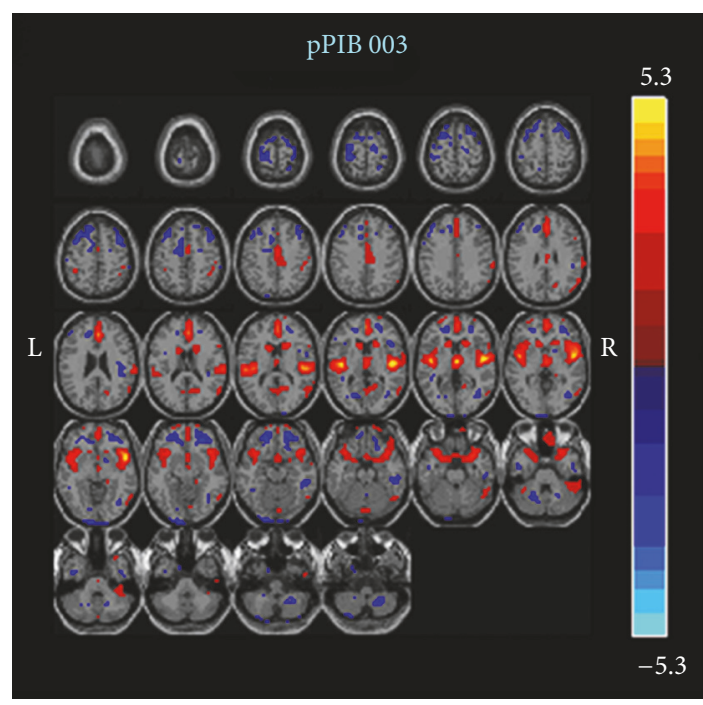

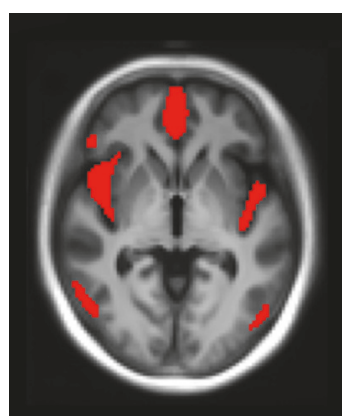

(b)

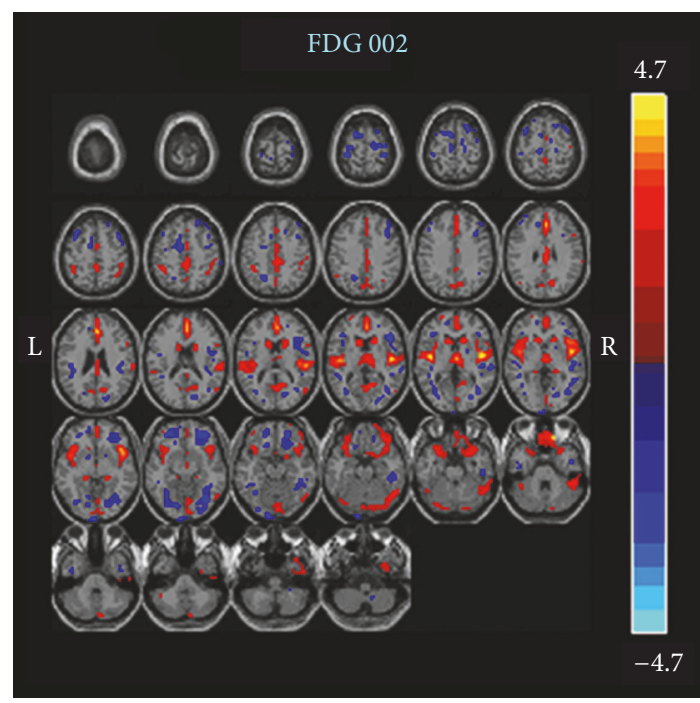

(c)

Figure 1: Correlated components of ${ }^{11} \mathrm{C}$-pPIB and ${ }^{18} \mathrm{~F}$-FDG. (a) Loading parameters with a significant correlation in all participants with ${ }^{18} \mathrm{~F}-\mathrm{FDG}$ (red) and ${ }^{11} \mathrm{C}$-pPIB (green). (b) Common regions of the correlated ${ }^{18} \mathrm{~F}-\mathrm{FDG}$ and ${ }^{11} \mathrm{C}$-pPIB components, including bilateral medial frontal gyrus, temporal lobe, and right insular lobe. (c) Correlated components of ${ }^{11} \mathrm{C}$-pPIB (left) and ${ }^{18} \mathrm{~F}-\mathrm{FDG}$ (right), including the medial frontal gyrus, anterior cingulate cortex, posterior cingulate cortex/precuneus, superior temporal gyrus, temporal pole, and orbitofrontal gyrus. ${ }^{11} \mathrm{C}$-pPIB, $\left({ }^{11} \mathrm{C}\right)$-labeled Pittsburgh Compound B; ${ }^{18}$ F-FDG, fludeoxyglucose F 18.

The comparison between $\mathrm{MCI}$ and $\mathrm{CN}$ patients within the correlated network revealed that the ${ }^{18} \mathrm{~F}$-FDG uptake was less in the rectal gyrus/Brodmann area 11 (BA11), BA40, left PCC, BA20, and inferior parietal lobule (IPL)/STG (Figure 3). In contrast, hypoperfusion was only detected in the IPL.

No statistically significant differences were observed in ${ }^{18} \mathrm{~F}-\mathrm{FDG}$ or PPIB data for $\mathrm{AD}$ and MCI patients.

\section{Discussion}

In contrast to earlier studies looking into the dual-features of dynamic PIB-PET and the similarities between ${ }^{11} \mathrm{C}$-pPIB and ${ }^{18}$ F-FDG images [4-6, 10, 24, 25], the present study identified functional brain networks from early-phase ${ }^{11} \mathrm{C}$-PIB data. We first used pICA to identify the brain networks from the ${ }^{11} \mathrm{C}$ pPIB-PET imaging data and then explored the discriminability of the brain networks in diagnostic group differences. Concomitantly we were able to evaluate the use of ${ }^{11} \mathrm{C}$-pPIB as a neurofunctional biomarker for $\mathrm{AD}$.
3.1. Highly Correlated Networks of ${ }^{11} C-p P I B$ and ${ }^{18} F-F D G$. It is well-documented that there are changes in the brain structure, function, and cognition in $\mathrm{AD}$ patients associated with changes in brain networks [26-29]. Using resting state functional connectivity MRI (rs-fMRI), both intra- and internetwork correlations have already been detected in $\mathrm{AD}$ patients. These mainly involved DMN, dorsal attention, salience, control, and sensory-motor networks [29, 30]. Although AD is associated with widespread disruption of functional connectivity, the DMN is generally affected the most. Specifically, a declined functional connectivity and hypometabolism were observed consistently using various methodologies [27-29]. In the present study, highly correlated brain networks of ${ }^{11} \mathrm{C}$-pPIB and ${ }^{18} \mathrm{~F}$-FDG data were identified using pICA. The correlated networks of ${ }^{18} \mathrm{~F}$-FDG and ${ }^{11} \mathrm{C}$-pPIB covered the MFG, ACC, PCC/precuneus, STG, temporal pole, and orbitofrontal gyrus and largely colocalized with the DMN $[22,23]$. The DMN regions are active at rest (hence the term "default") [22] but are less active during demanding cognitive tasks. Under physiological conditions, up to $80 \%$ of 
TABLE 3: ${ }^{18}$ F-FDG-revealed hypometabolic brain areas differentiating AD and CN groups.

\begin{tabular}{|c|c|c|c|c|c|c|}
\hline \multirow{2}{*}{ Brain region } & \multicolumn{3}{|c|}{ Voxel-level } & \multirow{2}{*}{$X$} & \multirow{2}{*}{$Y$} & \multirow{2}{*}{$Z$} \\
\hline & $T$ & $Z$ & $p_{\text {uncorrected }}$ & & & \\
\hline \multirow{2}{*}{ Limbic lobe/R ParaHippo } & 6.75 & 5.05 & 0.000 & 22 & -4 & -38 \\
\hline & 4.78 & 3.99 & 0.000 & 22 & 6 & -24 \\
\hline \multirow{2}{*}{ R STG/BA39 } & 6.40 & 4.88 & 0.000 & 58 & -58 & 26 \\
\hline & 5.18 & 4.23 & 0.000 & 54 & -54 & 44 \\
\hline \multirow{2}{*}{ R MTG/ ITG } & 5.99 & 4.68 & 0.000 & 54 & -64 & 18 \\
\hline & 5.85 & 4.60 & 0.000 & 66 & -40 & -14 \\
\hline Rectal gyrus & 5.89 & 4.62 & 0.000 & 8 & 32 & -26 \\
\hline Limbic lobe/L ParaHippo & 5.79 & 4.57 & 0.000 & -22 & 4 & -22 \\
\hline \multirow{2}{*}{ PCC/BA29 } & 5.64 & 4.49 & 0.000 & -2 & -42 & 20 \\
\hline & 5.51 & 4.41 & 0.000 & 0 & -26 & 32 \\
\hline \multirow{2}{*}{ R MFG/ACC } & 5.09 & 4.18 & 0.000 & 2 & 50 & -12 \\
\hline & 4.88 & 4.05 & 0.000 & 4 & 38 & 24 \\
\hline \multirow{2}{*}{ R SPL/BA7 } & 5.01 & 4.13 & 0.000 & 36 & -58 & 54 \\
\hline & 4.70 & 3.94 & 0.000 & 40 & -48 & 50 \\
\hline \multirow{2}{*}{ L STG/BA13 } & 4.95 & 4.10 & 0.000 & -50 & -4 & 0 \\
\hline & 4.42 & 3.76 & 0.000 & -46 & 6 & -2 \\
\hline \multirow{2}{*}{ L STG/BA42/Insula } & 4.79 & 4.00 & 0.000 & -64 & -36 & 18 \\
\hline & 3.82 & 3.36 & 0.000 & -54 & -32 & 18 \\
\hline L STG/BA38 & 4.73 & 3.96 & 0.000 & 26 & 6 & -48 \\
\hline \multirow{2}{*}{ L IFG/BA47 } & 4.70 & 3.94 & 0.000 & -40 & 24 & -16 \\
\hline & 4.09 & 3.55 & 0.000 & -38 & 14 & -12 \\
\hline
\end{tabular}

Threshold: $T=3.45, p=0.001$; Extent threshold: $k=50$ voxel, voxel size: [2.0, 2.0, 2.0] mm. ${ }^{18}$ F-FDG: fludeoxyglucose F 18; AD: Alzheimer's disease; CN: cognitively normal; STG: superior temporal gyrus; BA: Brodmann area; MTG: middle temporal gyrus; ITG: inferior temporal cortex; PCC: posterior cingulate cortex; MFG: medial frontal gyrus; ACC: anterior cingulate cortex; SPL: superior parietal lobe; IFG: inferior frontal gyrus.

TABLE $4:{ }^{11} \mathrm{C}$-pPIB-revealed hypoperfusion brain areas differentiating $\mathrm{AD}$ and $\mathrm{CN}$ groups.

\begin{tabular}{|c|c|c|c|c|c|c|}
\hline \multirow{2}{*}{ Brain region } & \multicolumn{3}{|c|}{ Voxel-level } & \multirow{2}{*}{$X$} & \multirow[t]{2}{*}{$Y$} & \multirow{2}{*}{$Z$} \\
\hline & $T$ & $Z$ & $p_{\text {uncorrected }}$ & & & \\
\hline \multirow{2}{*}{ R ITG/BA19 } & 5.66 & 4.50 & 0.000 & 54 & -64 & -6 \\
\hline & 5.32 & 4.31 & 0.000 & 54 & -66 & 14 \\
\hline L STG/BA22 & 5.00 & 4.13 & 0.000 & 66 & -46 & 6 \\
\hline Limbic lobe/L ParaHippo & 4.74 & 3.97 & 0.000 & -22 & 4 & -22 \\
\hline \multirow{2}{*}{ BA23/PCC } & 4.41 & 3.76 & 0.000 & 2 & -38 & 24 \\
\hline & 4.15 & 3.59 & 0.000 & -2 & -26 & 30 \\
\hline Insula/BA13 & 4.39 & 3.74 & 0.000 & 42 & -20 & 12 \\
\hline R STG/BA39 & 4.37 & 3.73 & 0.000 & 58 & -56 & 26 \\
\hline L STG/BA22 & 4.18 & 3.60 & 0.000 & -52 & 0 & 0 \\
\hline R ITG/BA20 & 3.89 & 3.41 & 0.000 & 58 & -8 & -34 \\
\hline ACC/BA24 & 3.86 & 3.38 & 0.000 & 2 & 32 & 16 \\
\hline Limbic lobe/R ParaHippo & 3.84 & 3.38 & 0.000 & 14 & -38 & -2 \\
\hline R SPL/BA7 & 3.82 & 3.35 & 0.000 & 36 & -58 & 54 \\
\hline R IPL/BA40 & 3.81 & 3.32 & 0.000 & 40 & -48 & 44 \\
\hline
\end{tabular}

Threshold: $T=3.45, p=0.001$; Extent threshold: $k=50$ voxel, Voxel size: $[2.0,2.0,2.0] \mathrm{mm} .{ }^{11} \mathrm{C}-\mathrm{pPIB}:\left({ }^{11} \mathrm{C}\right)$-labeled Pittsburgh Compound B; AD: Alzheimer's disease; CN: cognitively normal; ITG: inferior temporal cortex; STG: superior temporal gyrus; BA: Brodmann area; ACC: anterior cingulate cortex; SPL: superior parietal lobe; IPL: inferior parietal lobule. 


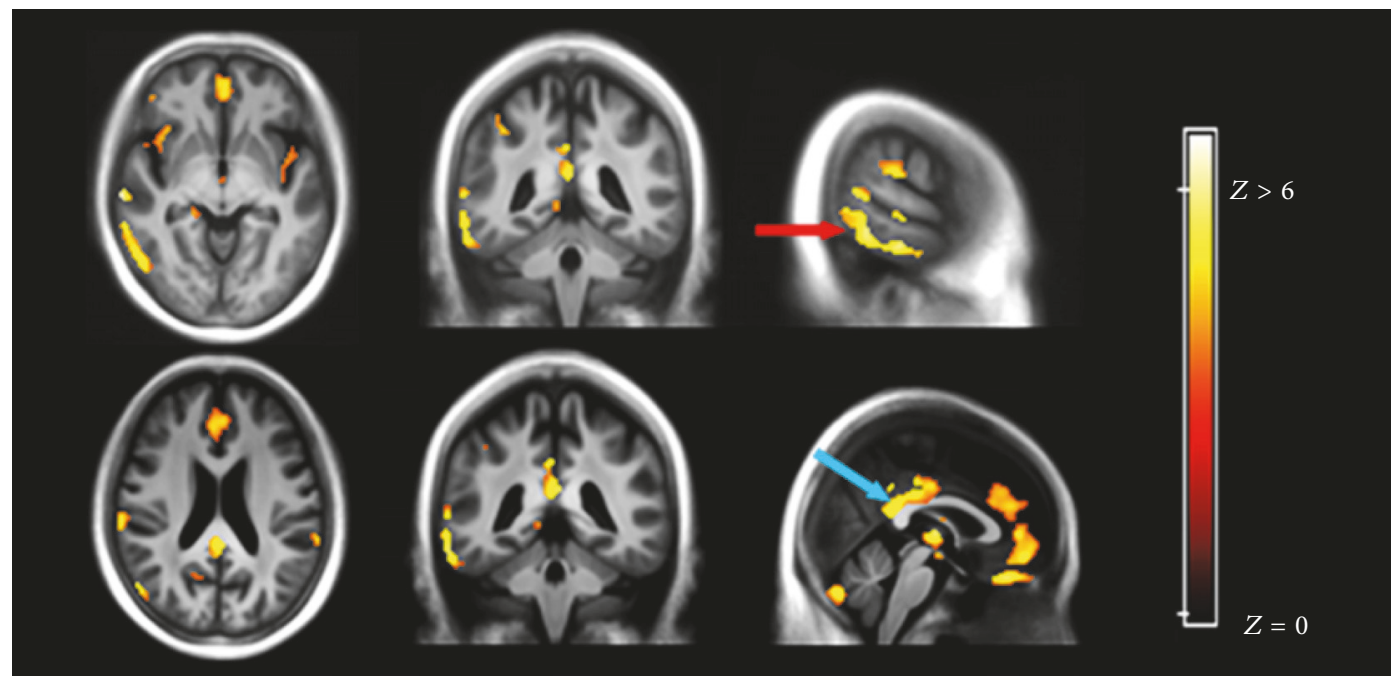

(a)

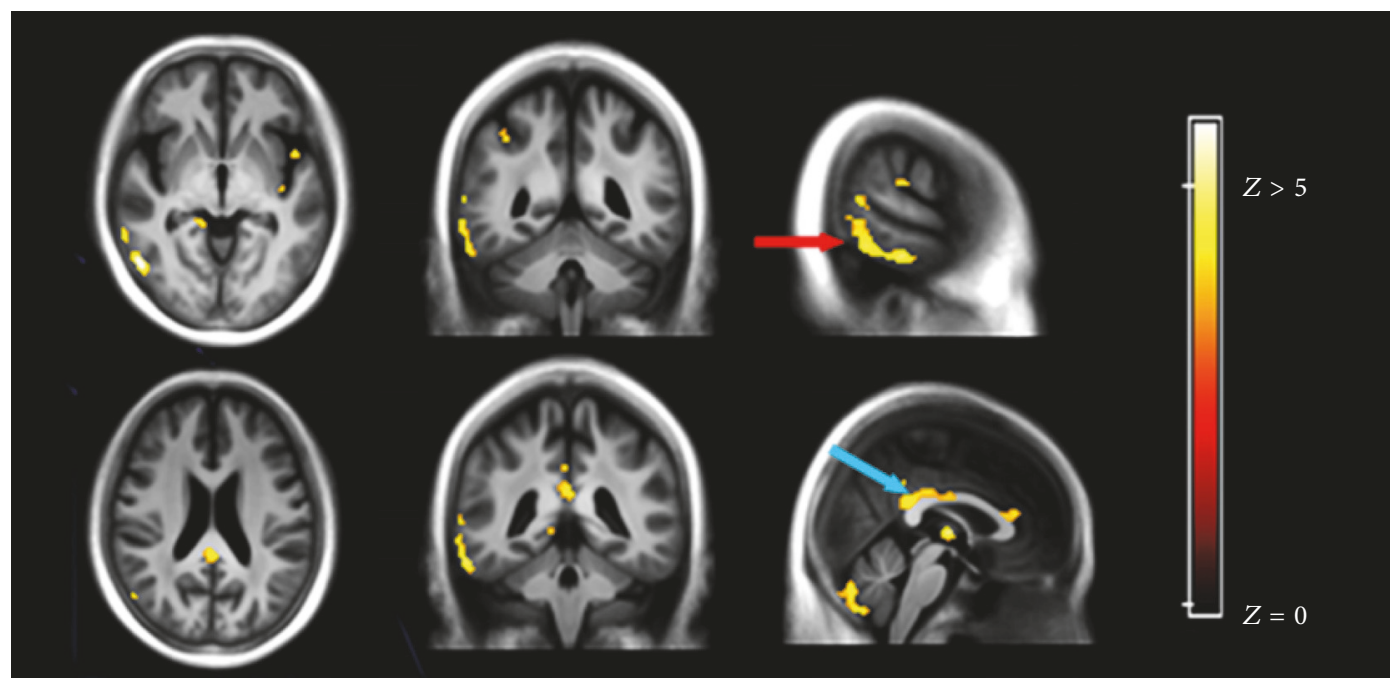

(b)

Figure 2: Comparison of ${ }^{18}$ F-FDG (a) and ${ }^{11} \mathrm{C}$-pPIB (b) between Alzheimer's disease (AD) and cognitively normal (CN) groups. Images show a similar decrease in the radioactive pattern, such as for the right superior temporal gyrus (red arrow, $x=-50, y=-4, z=5$ ) and posterior cingulate cortex (blue arrow, $x=2, y=-41, z=23) .{ }^{11} \mathrm{C}$-pPIB, $\left({ }^{11} \mathrm{C}\right.$ )-labeled Pittsburgh Compound B; ${ }^{18} \mathrm{~F}-\mathrm{FDG}$, fludeoxyglucose F 18.

the entire energy consumption by the brain at rest is spent on glutamate cycling, a biochemical process that can be observed by FDG-PET [31, 32]. In fact, the DMN is roughly divided into three major subdivisions, each with its own functional property: the ventral medial prefrontal cortex (supports emotional processing); the dorsal medial prefrontal cortex (self-referential mental activity); and the posterior cingulate cortex and adjacent precuneus plus the lateral parietal cortex (the recollection of prior experiences). These functional properties of DMN can be affected during task performance and also by various diseases [33]. For example, the episodic memory, requiring functional connectivity within the DMN [34, 35], is impaired in the early stages of AD. The abnormalities of DMN functional connectivity worsen with disease progression and are believed to explain the hypometabolism found in PET studies [36-39]. Moreover, FDG-PET measures both the CBF and the neuronal and synaptic activity [40]. A decrease in CBF is, furthermore, an indirect indicator of impairment caused by a decrease in the demand for blood. Nevertheless, the highly colocalized brain networks between glucose metabolism/rCBF and functional connectivity at rest indicated that glucose consumption and changes in $\mathrm{rCBF}$ are coupled and underpinning the neural activity. It is therefore argued that ${ }^{11} \mathrm{C}$-pPIB can be used in the future as a neurofunctional biomarker for neuroscience research.

3.2. Group Comparison of ${ }^{11} C-p P I B$ and ${ }^{18} F-F D G$ Measurements in Correlated Brain Networks. The second goal of this study was to evaluate whether the brain networks derived 


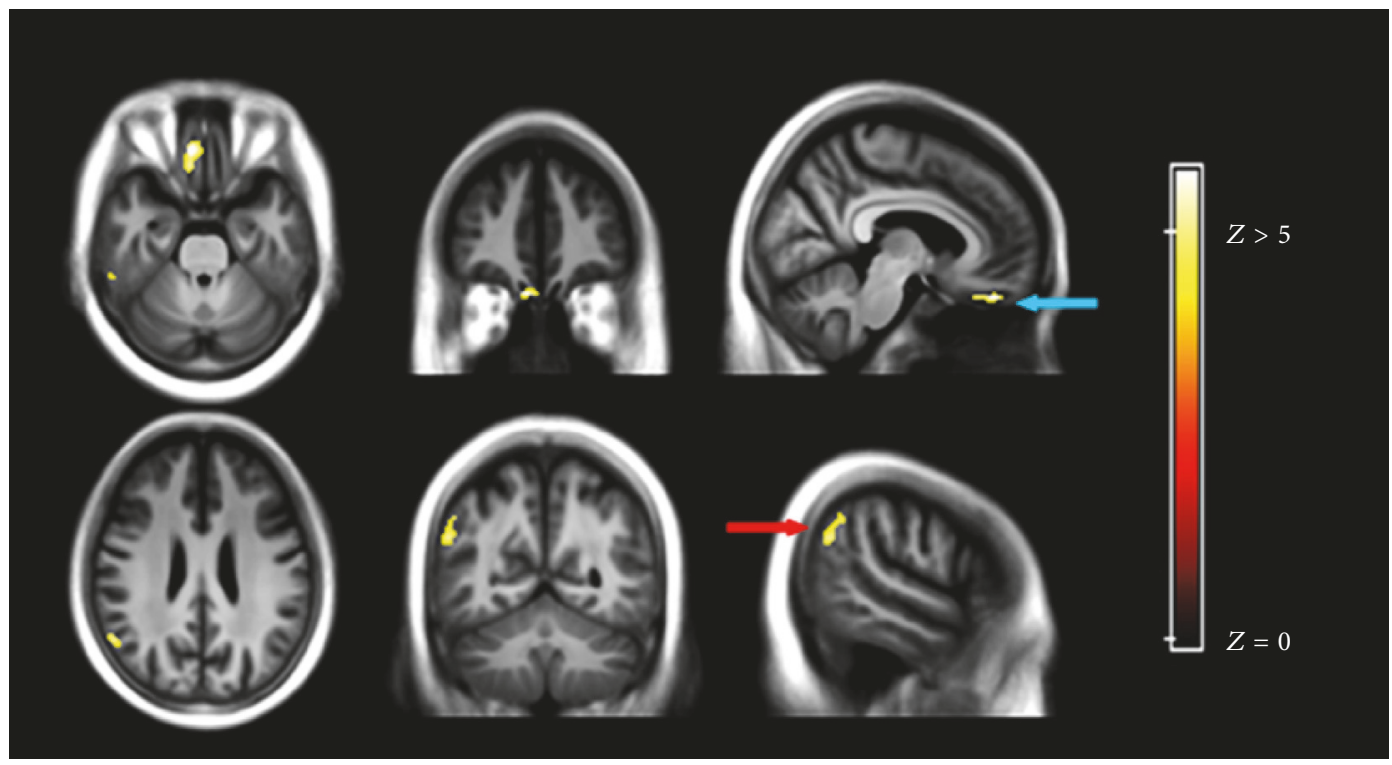

(a)

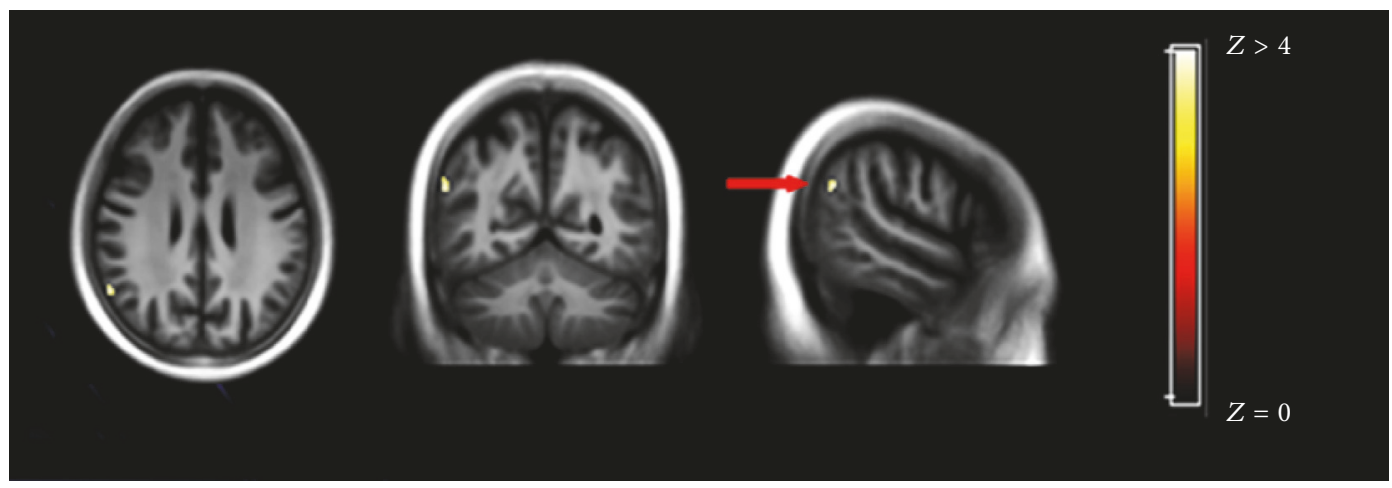

(b)

FIGURE 3: Comparison of ${ }^{18} \mathrm{~F}$-FDG (a) and ${ }^{11} \mathrm{C}$-pPIB (b) between mild cognitive impairment (MCI) and cognitively normal (CN) groups. (a) ${ }^{18}$ F-FDG images showing hypometabolic regions in the rectal gyrus (blue arrow, $x=8, y=42, z=-26$ ) and inferior parietal lobule (red arrow, $x=54, y=-52, z=50$ ) and (b) ${ }^{11} \mathrm{C}$-pPIB, showing only hypoperfusion in the inferior parietal lobule (red arrow, $x=58, y=-56$, $z=46) .{ }^{11} \mathrm{C}$-pPIB, $\left({ }^{11} \mathrm{C}\right)$-labeled Pittsburgh Compound B; ${ }^{18} \mathrm{~F}-\mathrm{FDG}$, fludeoxyglucose F 18.

from ${ }^{11} \mathrm{C}$-pPIB data could differentiate $\mathrm{AD} / \mathrm{MCI}$ patient groups from $\mathrm{CN}$ groups. Consistent with previous reports, $\mathrm{AD}$ patients were characteristically hypometabolic in the medial temporal lobe, STG, ACC, PCC/precuneus, SPL, and lateral temporoparietal cortex $[36,39] .{ }^{11} \mathrm{C}-\mathrm{pPIB}$ also revealed significant intergroup differences, with regions of hypoperfusion in the STG, inferior temporal gyrus, SPL, and PCC, which is consistent with previous studies using ${ }^{99 \mathrm{~m}}$ Technetium-single photon emission computed tomography showing specific patterns of hypoperfusion in parietaltemporal cortical areas [41, 42]. The comparison between MCI and $\mathrm{CN}$ patients revealed hypometabolism in the rectal gyrus/BA11, IPL, left PCC, BA20, and IPL/STG, but only the IPL was detected by ${ }^{11} \mathrm{C}$-pPIB. The most reliable, early changes in metabolism are believed to be seen in the PCC $[24,36]$. In fact, the IPL is an important region of the DMN and has a close functional connection with the
PCC/precuneus [43, 44]. Esposito et al. reported that MCI patients who convert to $\mathrm{AD}$ showed increased connectivity in the right IPL, suggesting that this region plays an active role in the AD process [45]. Arguably, the hypometabolism and hyperperfusion that was detected in the IPL in this study was indicative of early neurodegeneration in $\mathrm{AD}$. No significant difference was detected by either ${ }^{18}$ F-FDG or ${ }^{11} \mathrm{C}$ pPIB when comparing AD and MCI groups in the current study. We support our observations as follows. Firstly, the AD patients enrolled in the study who received the dual-tracer PET scan had slight to mild dementia. In addition, despite the amnestic type of the recruited MCI patients, MCI is still a heterogeneous syndrome and subject to various pathological substrates and clinical progress [46]. Therefore, the ${ }^{18}$ F-FDG and ${ }^{11} \mathrm{C}$-pPIB data may not detect a difference between $\mathrm{AD}$ and MCI due to the differences in clinical symptom severity and the heterogeneity in MCI. Secondly, the MCI patients 
were significantly older than the AD patients. Although age was regressed in the data analysis and no obvious vascular diseases were found on MR images of all subjects, the CBF will be affected by atherosclerosis, which usually progresses with aging. Thus, both patient's age and its possibly associated reduced CBF may have obscured our results. As discussed, the brain network of ${ }^{11} \mathrm{C}$-pPIB was found highly colocalized with $\mathrm{DMN}$ in $\mathrm{AD}$ pathology in this study. It is argued that disease-specific alternations of the brain networks can be detected by ${ }^{11} \mathrm{C}$-pPIB corresponding to distinctive pathophysiological processes, which merits further studies.

One limitation of the present study is the relatively small sample size. More recruits would give a larger dataset, which might help to identify the fine-grained brain networks underpinning ${ }^{11} \mathrm{C}$-pPIB and ${ }^{18}$ F-FDG. Furthermore, only the typical $\mathrm{AD}$ and amnestic MCI patients were enrolled in the current study and their disrupted brain networks mainly involved the DMN [27, 28, 47]. However, AD patients with atypical symptoms, such as Logopenic primary progressive aphasia and posterior cortical atrophy, had language and visual brain network dysfunctions matching their clinical symptoms $[19,47] .{ }^{11} \mathrm{C}$-pPIB might, therefore, be used to validate the syndrome-specific alterations of the brain network in these clinical variants of $\mathrm{AD}$. In addition, the present study did not investigate the functional networks derived from rsfMRI data of the same cohort. Therefore, it is impossible to directly correlate the networks of ${ }^{11} \mathrm{C}$-pPIB and ${ }^{18} \mathrm{~F}-\mathrm{FDG}$ with the functional networks in rs-fMRI data.

\section{Conclusions}

Here, we explored the ${ }^{11} \mathrm{C}$-pPIB spatial distribution pattern in $\mathrm{AD}, \mathrm{MCI}$, and $\mathrm{CN}$ patients. The pICA results revealed that the hypoperfusion pattern detected by ${ }^{11} \mathrm{C}$-pPIB was in agreement with the hypometabolism reflected by ${ }^{18} \mathrm{~F}-\mathrm{FDG}$, both of which were colocalized with the DMN. These results validated that ${ }^{11} \mathrm{C}$-pPIB could be a reliable biomarker of neural function and provide complementary information for ${ }^{18}$ F-FDG examination in AD.

\section{Materials and Methods}

5.1. Eligibility and Study Design. The study cohort was the same as in our previous studies $[10,25]$ and included $14 \mathrm{AD}$, $12 \mathrm{MCI}$, and $14 \mathrm{CN}$ patients. The study was approved by the institutional review board of the Chinese PLA General Hospital. The study was compliant with the principles of the Declaration of Helsinki. All participants, or their appropriate representatives, signed informed consent forms after receiving a comprehensive written and verbal description of the study.

5.2. PET/CT Imaging. All patients underwent ${ }^{11} \mathrm{C}-\mathrm{PIB}$ and ${ }^{18}$ F-FDG scanning in a random order within two weeks. PET/CT scanning was performed using a Biograph Truepoint 64 (Siemens Healthcare, Germany) consisting of a PET scanner and a multislice CT. A vacuum cushion was used to restrict the participant's head to minimize movement during the scanning.
${ }^{11} \mathrm{C}$-PIB was synthesized from its corresponding precursors as described elsewhere [48]. In brief, ${ }^{11} \mathrm{C}-\mathrm{PIB}$ was synthesized by bubbling the ${ }^{11} \mathrm{CH} 3$-Triflate through an acetone solution of 6-OH-BTA-0. It was then purified by semipreparative HPLC and reformulated with a radiochemical purity of $>95 \%$ and a specific activity of $50 \mathrm{GBq}^{-1} \mathrm{~mol}^{-1}$ $\left(1.48 \mathrm{Ci} \mu \mathrm{mol}^{-1}\right)$. The protocol for ${ }^{11} \mathrm{C}$-PIB scanning included an initial CT acquisition with intravenous tracer injection, followed by an immediate dynamic PET scan. A spiral CT for the brain was acquired with CT parameters of $120 \mathrm{kV}$, $100 \mathrm{~mA}$, and slice thickness $3.75 \mathrm{~mm}$, equal to that of PET. Then, a dynamic PET emission scan in $3 \mathrm{D}$ acquisition mode was started simultaneously with a single intravenous bolus of ${ }^{11} \mathrm{C}$-PIB at $4.81-5.55 \mathrm{MBq}(0.13-0.15 \mathrm{mCi}) \mathrm{kg}^{-1}$. Dynamic brain PET images were collected continuously for $60 \mathrm{~min}$, and the data were binned into 26 frames $(1 \times 10 \mathrm{sec}, 6 \times 5 \mathrm{sec}$, $4 \times 20 \mathrm{sec}, 2 \times 1 \mathrm{~min}, 3 \times 2 \mathrm{~min}$, and $10 \times 5 \mathrm{~min})$.

${ }^{18}$ F-FDG-PET/CT scans were obtained $50 \mathrm{~min}$ after an intravenous injection of ${ }^{18} \mathrm{~F}-\mathrm{FDG}$ at 4.81 to $5.55 \mathrm{MBq}$ (0.13-0.15 mCi) $\mathrm{kg}^{-1}$. All participants were instructed to fast for 4 to $6 \mathrm{~h}$. The blood glucose levels were also measured before injection to ensure that the levels were within the reference range. A 5-min frame was collected in 3D acquisition mode. Data obtained from the CT scans were used to correct the attenuation for PET emission data.

5.3. MR Structural Imaging. All participants underwent structural MRI with a 3.0-T GE scanner (Signa HD, WI, USA) and a standard GE quadrature head coil. The MRI and PET/CT examinations were performed within one week. The scan protocol included a high-resolution 3D T1-weighted spoiled gradient recalled echo sequence $(\mathrm{TR}=7.0 \mathrm{~ms}, \mathrm{TE}=$ $2.9 \mathrm{~ms}$, Inversion time $=450 \mathrm{~ms}$, thickness $=1.2 \mathrm{~mm}$, matrix $=256 \times 256, \mathrm{FOV}=240 \mathrm{~mm}$, and in plane resolution $=0.9 \times$ $0.9 \mathrm{~mm}^{2}$ ) to produce contiguous sagittal anatomic images for subsequent spatial normalization and coregistration.

The preprocessing of MRI and PET imaging is detailed elsewhere [10]. Specifically, all structural MRI images were segmented into gray matter, white matter, and cerebrospinal fluid and then used to construct a population template using DARTEL of SPM8 (http://www.fil.ion.ucl.ac.uk/spm). The mid-frame (the 16th frame) of the dynamic PIB images and FDG images was coregistered with the corresponding MRI scan, and the PET scans were transformed to the population template with the deformation fields generated in the registration procedure of the MRI scans. Finally, all images were spatially normalized to the Montreal Neurological Institute space.

5.4. Computation of ${ }^{11} C-p P I B$ Image from Dynamic ${ }^{11} C-P I B$ Scans. The procedure to compute the ${ }^{11} \mathrm{C}$-pPIB images from the dynamic PIB scans has been reported previously [10]. Particularly, ${ }^{11} \mathrm{C}$-pPIB images were computed based on a 7-min time-window of the dynamic ${ }^{11} \mathrm{C}$-PIB-PET, starting from 9th frame to 15th frame and corresponding to the frames of 1.33-8 min, yielding the highest correlation between ${ }^{18} \mathrm{~F}-\mathrm{FDG}$ and ${ }^{11} \mathrm{C}-\mathrm{PIB}(R=0.87)$. Also, ${ }^{11} \mathrm{C}-\mathrm{PIB}$ and ${ }^{18} \mathrm{~F}-\mathrm{FDG}$ images shared a similar radioactive distribution pattern in $\mathrm{CN}, \mathrm{MCI}$, and $\mathrm{AD}$ groups. 
5.5. Statistical Analysis. The pICA algorithm (Fusion ICA Toolbox, http://icatb.sourceforge.net, with MATLAB 7.1) was applied to the ${ }^{11} \mathrm{C}$-pPIB and 18F-FDG images of all the patients to jointly extract statistical factors from the 11C-pPIB and 18F-FDG data and identify their mutual relationship.

The number of independent components was set to eight, based on a previous study [49]. The outputs from the pICA were pairs of ${ }^{11} \mathrm{C}$-pPIB and ${ }^{18} \mathrm{~F}$-FDG independent components. Their correlation coefficients were indicative of a relationship between the two modalities. The independent components of ${ }^{11} \mathrm{C}$-pPIB and ${ }^{18} \mathrm{~F}$-FDG data measured the perfusion and metabolic variability among the participants. All components had a threshold at a supra level, $|Z|>1.5$, to identify statistically significant regions that contributed to the overall signal of the corresponding components. Correlation coefficients between components of the two imaging modalities were used to identify the most associated pairs of components.

Subsequently, voxel wise ${ }^{11} \mathrm{C}$-pPIB and ${ }^{18}$ F-FDG measurements within the most associated pair of components were compared between $\mathrm{AD}-\mathrm{CN}, \mathrm{MCI}-\mathrm{CN}$, and $\mathrm{AD}-\mathrm{MCI}$ groups using a two-sample $t$-test. The multiple comparisons were corrected for using the AlphaSim program in REST (http://restfmri.net/forum/rest) with a full-width at halfmaximum of $6 \mathrm{~mm}$. The threshold for the group differences was $p<0.05$ with the AlphaSim correction (with $p<0.001$ threshold and a minimum cluster size of 12 voxels).

\section{Disclosure}

An earlier version of this work was presented as a poster at the Annual Congress of the European Association of Nuclear Medicine (EANM'17 CME Sessions), 2017.

\section{Conflicts of Interest}

The authors declare that they have no conflicts of interest.

\section{Authors' Contributions}

Liping Fu and Linwen Liu contributed equally to this work.

\section{Acknowledgments}

The authors would like to thank Xi Zhang, Bo Zhou, Zengqiang Zhang, and Pan Wang for patient recruitment; Dayi Yin, Jiajin Liu, and Can Li for technical support; and Xiaojun Zhang and Jian Liu for helping with the PET radiochemistry. This study was sponsored by the Central Health Care Research W2013BJ14 (to Baixuan Xu), National Major Scientific Instruments and Equipment Development Projects 2011 YQ030114 (to Jiahe Tian), and the China Postdoctoral Science Foundation 20090461433 and 2013M542515 (to Liping $\mathrm{Fu}$ ).

\section{References}

[1] G. M. McKhann, D. S. Knopman, H. Chertkow et al., "The diagnosis of dementia due to Alzheimer's disease: Recommendations from the National Institute on Aging-Alzheimer's Association workgroups on diagnostic guidelines for Alzheimer's disease," Alzheimer's \& Dementia, vol. 7, no. 3, pp. 263-269, 2011.

[2] T. Nihashi, H. Yatsuya, K. Hayasaka et al., "Direct comparison study between FDG-PET and IMP-SPECT for diagnosing Alzheimer's disease using 3D-SSP analysis in the same patients," Radiation Medicine - Medical Imaging and Radiation Oncology, vol. 25, no. 6, pp. 255-262, 2007.

[3] O. B. Paulson, S. G. Hasselbalch, E. Rostrup, G. M. Knudsen, and D. Pelligrino, "Cerebral blood flow response to functional activation," Journal of Cerebral Blood Flow \& Metabolism, vol. 30, no. 1, pp. 2-14, 2010.

[4] A. Forsberg, H. Engler, G. Blomquist, B. Långström, and A. Nordberg, "The use of PIB-PET as a dual pathological and functional biomarker in AD," Biochimica et Biophysica Acta (BBA) - Molecular Basis of Disease, vol. 1822, no. 3, pp. 380-385, 2012.

[5] A. H. Rostomian, C. Madison, G. D. Rabinovici, and W. J. Jagust, "Early 11C-PIB frames and 18F-FDG PET measures are comparable: A study validated in a cohort of AD and FTLD patients," Journal of Nuclear Medicine, vol. 52, no. 2, pp. 173-179, 2011.

[6] E. Rodriguez-Vieitez, S. F. Carter, K. Chiotis et al., "Comparison of early-phase 11C-Deuterium-L-Deprenyl and 11C-Pittsburgh Compound B PET for Assessing Brain Perfusion in Alzheimer Disease," Journal of Nuclear Medicine, vol. 57, no. 7, pp. 10711077, 2016.

[7] S. Daerr, M. Brendel, C. Zach et al., "Evaluation of early-phase [18F]-florbetaben PET acquisition in clinical routine cases," NeuroImage: Clinical, vol. 14, pp. 77-86, 2017.

[8] S. Tiepolt, S. Hesse, M. Patt et al., "Early [18F]florbetaben and [11C]PiB PET images are a surrogate biomarker of neuronal injury in Alzheimer's disease," European Journal of Nuclear Medicine and Molecular Imaging, vol. 43, no. 9, pp. 1700-1709, 2016.

[9] E. Rodriguez-Vieitez, A. Leuzy, K. Chiotis, L. Saint-Aubert, A. Wall, and A. Nordberg, "Comparability of [18F]THK5317 and [11C]PIB blood flow proxy images with [18F]FDG positron emission tomography in Alzheimer's disease," Journal of Cerebral Blood Flow \& Metabolism, vol. 37, no. 2, pp. 740-749, 2016.

[10] L. Fu, L. Liu, J. Zhang, B. Xu, Y. Fan, and J. Tian, "Comparison of dual-biomarker PIB-PET and dual-tracer PET in AD diagnosis," European Radiology, vol. 24, no. 11, pp. 2800-2809, 2014.

[11] M. J. McKeown, T.-P. Jung, S. Makeig et al., "Spatially independent activity patterns in functional MRI data during the Stroop color-naming task," Proceedings of the National Acadamy of Sciences of the United States of America, vol. 95, no. 3, pp. 803810, 1998.

[12] R. Vigário, J. Särelä, V. Jousmiki, M. Hämäläinen, and E. Oja, "Independent component approach to the analysis of EEG and MEG recordings," IEEE Transactions on Biomedical Engineering, vol. 47, no. 5, pp. 589-593, 2000.

[13] E. Milne, A. Scope, O. Pascalis, D. Buckley, and S. Makeig, "Independent Component Analysis Reveals Atypical Electroencephalographic Activity During Visual Perception in Individuals with Autism," Biological Psychiatry, vol. 65, no. 1, pp. 22-30, 2009. 
[14] R. Grandchamp, C. Braboszcz, S. Makeig, and A. Delorme, "Stability of ICA decomposition across within-subject EEG datasets., Conference proceedings: IEEE Engineering in Medicine and Biology Society, vol. 2012, pp. 6735-6739, 2012.

[15] L. Xu, K. M. Groth, G. Pearlson, D. J. Schretlen, and V. D. Calhoun, "Source-based morphometry: The use of independent component analysis to identify gray matter differences with application to schizophrenia," Human Brain Mapping, vol. 30, no. 3, pp. 711-724, 2009.

[16] H.-J. Park, J.-J. Kim, T. Youn, D. S. Lee, M. C. Lee, and J. S. Kwon, "Independent component model for cognitive functions of multiple subjects using [15O]H2O PET images," Human Brain Mapping, vol. 18, no. 4, pp. 284-295, 2003.

[17] V. D. Calhoun, T. Adali, K. A. Kiehl, R. Astur, J. J. Pekar, and G. D. Pearlson, "A method for multitask fMRI data fusion applied to schizophrenia," Human Brain Mapping, vol. 27, no. 7, pp. 598610, 2006.

[18] D. Tosun, N. Schuff, L. M. Shaw, J. Q. Trojanowski, and M. W. Weiner, "Relationship between CSF biomarkers of Alzheimer's disease and rates of regional cortical thinning in ADNI data," Journal of Alzheimer's Disease, vol. 26, supplement 3, pp. 77-90, 2011.

[19] R. Laforce Jr., D. Tosun, P. Ghosh et al., "Parallel ICA of FDG-PET and PiB-PET in three conditions with underlying Alzheimer's pathology," NeuroImage: Clinical, vol. 4, pp. 508$516,2014$.

[20] A. Drzezga, J. A. Becker, K. R. A. Van Dijk et al., "Neuronal dysfunction and disconnection of cortical hubs in non-demented subjects with elevated amyloid burden," Brain, vol. 134, no. 6, pp. 1635-1646, 2011.

[21] N. Myers, L. Pasquini, J. Göttler et al., "Within-patient correspondence of amyloid- $\beta$ and intrinsic network connectivity in Alzheimer's disease," Brain, vol. 137, no. 7, pp. 2052-2064, 2014.

[22] M. E. Raichle, A. M. MacLeod, A. Z. Snyder, W. J. Powers, D. A. Gusnard, and G. L. Shulman, "A default mode of brain function," Proceedings of the National Acadamy of Sciences of the United States of America, vol. 98, no. 2, pp. 676-682, 2001.

[23] R. L. Buckner, J. R. Andrews-Hanna, and D. L. Schacter, "The brain's default network: anatomy, function, and relevance to disease," Annals of the New York Academy of Sciences, vol. 1124, pp. 1-38, 2008.

[24] A. Kadir, O. Almkvist, A. Forsberg et al., "Dynamic changes in PET amyloid and FDG imaging at different stages of Alzheimer's disease," Neurobiology of Aging, vol. 33, no. 1, pp. 198-e14, 2012.

[25] L. Liu, L. Fu, X. Zhang et al., "Combination of dynamic ${ }^{11} \mathrm{C}$ PIB PET and structural MRI improves diagnosis of Alzheimer's disease," Psychiatry Research: Neuroimaging, vol. 233, no. 2, pp. 131-140, 2015.

[26] R. L. Buckner, A. Z. Snyder, B. J. Shannon et al., "Molecular, structural, and functional characterization of Alzheimer's disease: evidence for a relationship between default activity, amyloid, and memory," The Journal of Neuroscience, vol. 25, no. 34, pp. 7709-7717, 2005.

[27] W. W. Seeley, R. K. Crawford, J. Zhou, B. L. Miller, and M. D. Greicius, "Neurodegenerative diseases target large-scale human brain networks," Neuron, vol. 62, no. 1, pp. 42-52, 2009.

[28] J. Zhou, E. D. Gennatas, J. H. Kramer, B. L. Miller, and W. W. Seeley, "Predicting Regional Neurodegeneration from the Healthy Brain Functional Connectome," Neuron, vol. 73, no. 6, pp. 1216-1227, 2012.
[29] E. L. Dennis and P. M. Thompson, "Functional brain connectivity using fMRI in aging and Alzheimer's disease," Neuropsychology Review, vol. 24, no. 1, pp. 49-62, 2014.

[30] M. R. Brier, J. B. Thomas, A. Z. Snyder et al., "Loss of intranetwork and internetwork resting state functional connections with Alzheimer's disease progression," The Journal of Neuroscience, vol. 32, no. 26, pp. 8890-8899, 2012.

[31] R. G. Shulman, D. L. Rothman, K. L. Behar, and F. Hyder, "Energetic basis of brain activity: Implications for neuroimaging," Trends in Neurosciences, vol. 27, no. 8, pp. 489-495, 2004.

[32] N. R. Sibson, A. Dhankhar, G. F. Mason, K. L. Behar, D. L. Rothman, and R. G. Shulman, "In vivo 13C NMR measurements of cerebral glutamine synthesis as evidence for glutamateglutamine cycling," Proceedings of the National Acadamy of Sciences of the United States of America, vol. 94, no. 6, pp. 26992704, 1997.

[33] M. E. Raichle, "The brain's default mode network," Annual Review of Neuroscience, vol. 38, pp. 433-447, 2015.

[34] R. L. Buckner, "Memory and executive function in aging and ad: Multiple factors that cause decline and reserve factors that compensate," Neuron, vol. 44, no. 1, pp. 195-208, 2004.

[35] M. W. Cole, S. Pathak, and W. Schneider, "Identifying the brain's most globally connected regions," NeuroImage, vol. 49, no. 4, pp. 3132-3148, 2010.

[36] S. Minoshima, B. Giordani, S. Berent, K. A. Frey, N. L. Foster, and D. E. Kuhl, "Metabolic reduction in the posterior cingulate cortex in very early Alzheimer's disease," Annals of Neurology, vol. 42, no. 1, pp. 85-94, 1997.

[37] D. Zhang, A. Z. Snyder, J. S. Shimony, M. D. Fox, and M. E. Raichle, "Noninvasive functional and structural connectivity mapping of the human thalamocortical system," Cerebral Cortex, vol. 20, no. 5, pp. 1187-1194, 2010.

[38] J. Zhou, M. D. Greicius, E. D. Gennatas et al., "Divergent network connectivity changes in behavioural variant frontotemporal dementia and Alzheimer's disease," Brain, vol. 133, no. 5, pp. 1352-1367, 2010.

[39] A. Caroli, A. Prestia, K. Chen et al., "Summary metrics to assess Alzheimer disease-related hypometabolic pattern with 18F-FDG PET: Head-to-head comparison," Journal of Nuclear Medicine, vol. 53, no. 4, pp. 592-600, 2012.

[40] L. Sokoloff, "Relationships among local functional activity, energy metabolism, and blood flow in the central nervous system," Federation Proceedings, vol. 40, no. 8, pp. 2311-2316, 1981.

[41] H. Hanyu, S. Abe, H. Arai, T. Asano, T. Iwamoto, and M. Takasaki, "Diagnostic accuracy of single photon emission computed tomography in Alzheimer's disease," Gerontology, vol. 39, no. 5, pp. 260-266, 1993.

[42] J. T. O’Brien, S. Eagger, G. M. S. Syed, B. J. Sahakian, and R. Levy, "A study of regional cerebral blood flow and cognitive performance in Alzheimer's disease," Journal of Neurology, Neurosurgery \& Psychiatry, vol. 55, no. 12, pp. 1182-1187, 1992.

[43] B. Ćurčić-Blake, L. van der Meer, G. H. M. Pijnenborg, A. S. David, and A. Aleman, "Insight and psychosis: Functional and anatomical brain connectivity and self-reflection in Schizophrenia," Human Brain Mapping, vol. 36, no. 12, pp. 4859-4868, 2015.

[44] H.-J. Li, X. Nie, H.-H. Gong, W. Zhang, S. Nie, and D.-C. Peng, "Abnormal resting-state functional connectivity within the default mode network subregions in male patients with obstructive sleep APNEA," Neuropsychiatric Disease and Treatment, vol. 12, article no. A23, pp. 203-212, 2016. 
[45] R. Esposito, A. Mosca, V. Pieramico, F. Cieri, N. Cera, and S. L. Sensi, "Characterization of resting state activity in MCI individuals," PeerJ, vol. 2013, no. 1, article no. e135, 2013.

[46] M. S. Albert, S. T. DeKosky, D. Dickson et al., "The diagnosis of mild cognitive impairment due to Alzheimer's disease: recommendations from the National Institute on AgingAlzheimer's Association workgroups on diagnostic guidelines for Alzheimer's disease," Alzheimer's \& Dementia, vol. 7, no. 3, pp. 270-279, 2011.

[47] M. Lehmann, P. M. Ghosh, C. Madison et al., "Diverging patterns of amyloid deposition and hypometabolism in clinical variants of probable Alzheimer's disease," Brain, vol. 136, no. 3, pp. 844-858, 2013.

[48] C. Philippe, D. Haeusler, M. Mitterhauser et al., "Optimization of the radiosynthesis of the Alzheimer tracer 2-(4$\mathrm{N}$-[11C]methylaminophenyl)-6-hydroxybenzothiazole ([11C]PIB)," Applied Radiation and Isotopes, vol. 69, no. 9, pp. 1212-1217, 2011.

[49] S. A. Meda, B. Narayanan, J. Liu et al., "A large scale multivariate parallel ICA method reveals novel imaging-genetic relationships for Alzheimer's disease in the ADNI cohort," NeuroImage, vol. 60, no. 3, pp. 1608-1621, 2012. 


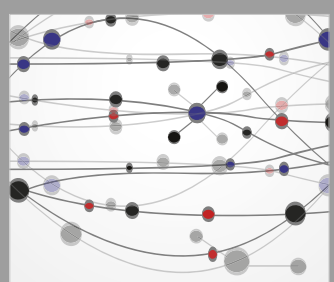

The Scientific World Journal
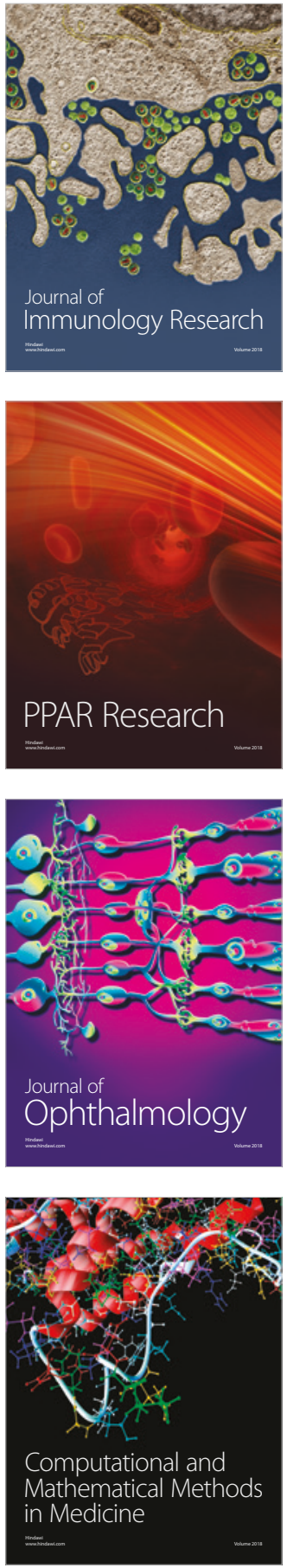

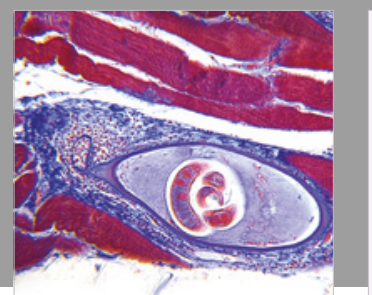

Gastroenterology Research and Practice

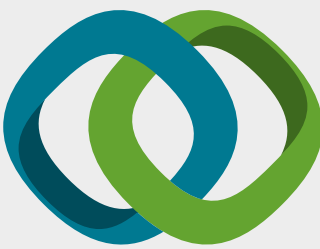

\section{Hindawi}

Submit your manuscripts at

www.hindawi.com
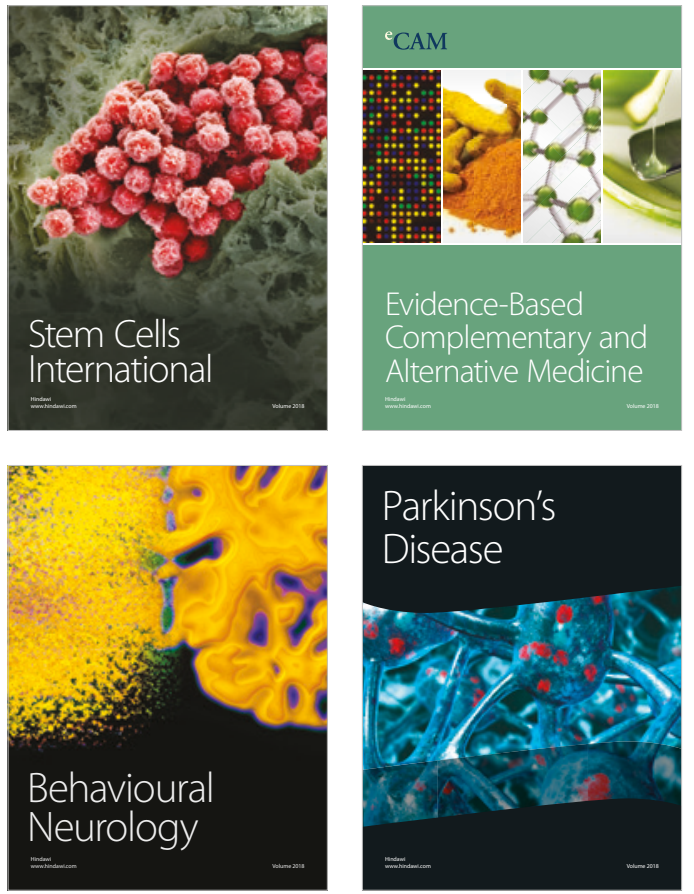

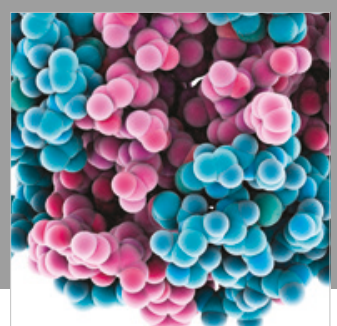

ournal of

Diabetes Research

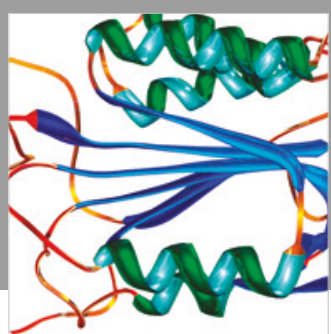

Disease Markers
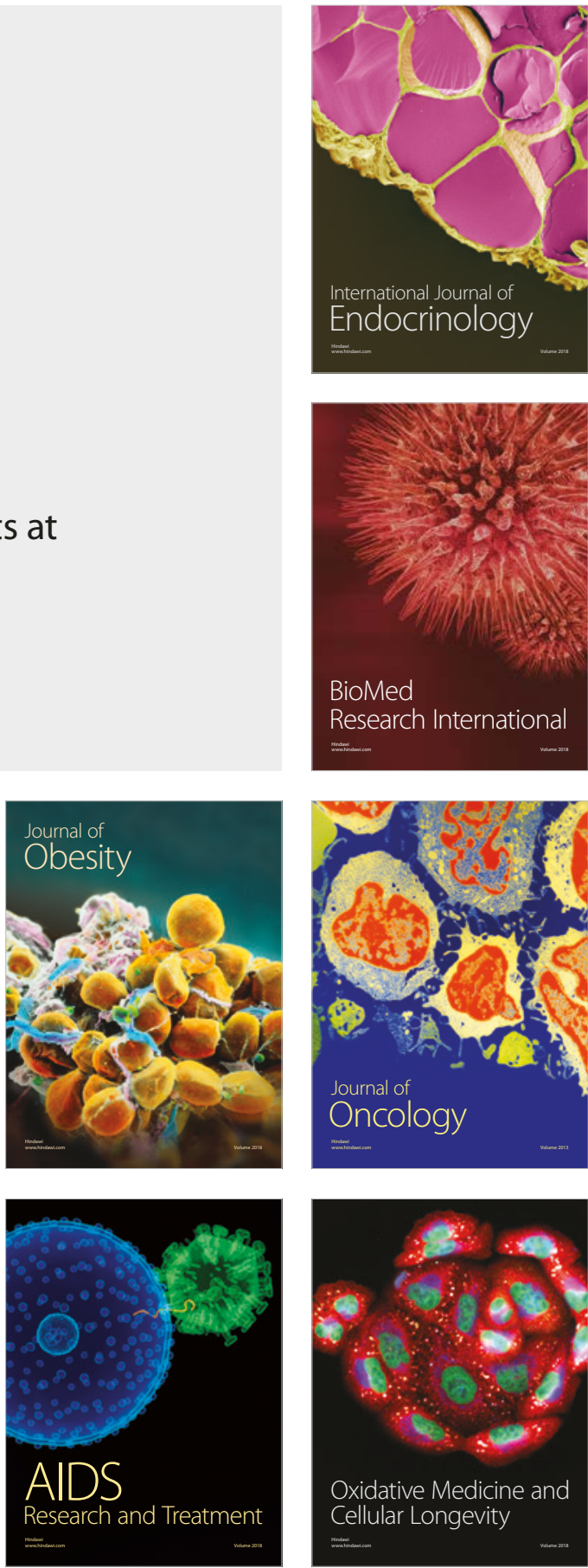